\title{
Association of alcohol consumption and components of metabolic syndrome among people in rural China
}

Jing Xiao ${ }^{1}$, Jian-Ping Huang ${ }^{2}$, Guang-Fei Xu ${ }^{3}$, De-Xi Chen ${ }^{1}$, Gui-Yun Wu ${ }^{1}$, Min Zhang ${ }^{1}$, Yi Shen ${ }^{1}$ and Hui Cai ${ }^{{ }^{*}}$

\begin{abstract}
Background: Accumulative evidence in the literature suggests alcohol consumption is a protective factor of the metabolic syndrome (MS). However, few studies investigated the relationship between alcohol consumption and components of MS. We examined association of several types of alcoholic beverage with components of MS among people in rural China.
\end{abstract}

Methods: In the Nantong Metabolic Syndrome Study (NMSS), a cross-sectional study, a total of 20,502 participants, including 13,505 women and 6,997 men aged 18-74 years, were recruited between 2007 and 2008 in Nantong, China. Socio-economic status, dietary intake, physical exercise, alcoholic beverage consumption, and smoking status information were obtained, and triglycerides (TG), high-density lipoprtein cholesterol (HDL-c), blood pressure (BP) and blood glucose level were examined for all participants. Logistic regression model and the restricted cubic spline approach were used to analyze the associations between alcoholic beverage consumption and MS components.

Results: The MS prevalence was $21.1 \%$ in the whole population, which was significantly low among drinkers (20.6\%), compared with non-drinkers (23.6\%) in women, and was comparable in men (16.4\% versus 17.1\%). High $\mathrm{HDL}-\mathrm{c}$ level was observed among drinkers, compared with non-drinkers in both men and women. Low TG level and Systolic BP (SBP) were found only among rice wine drinkers in women, and high waist circumference, high TG and $\mathrm{BP}$ were found among beer and liquor drinkers in men. Furthermore, we found that the highest quartile of rice wine drink in women may decrease $24 \%$ risk of high TG, $30 \%$ risk of low $\mathrm{HDL}-\mathrm{c}$ and $43 \%$ risk of high glucose among MS components cases respectively, compared with non-drinkers ( $p$ for trend $<0.01$ for those three components). While compared non-drinkers among men, the highest quartile of liquor drink may increase 32\% risk of high SBP, 55\% risk of high Diastolic BP (DBP) and 34\% risk of abdominal obesity among MS components cases respectively, but decrease $45 \%$ risk of low HDL-c ( $p$ for trend $<0.05$ for those four components).

Conclusion: Our data suggested that all alcoholic beverages increased HDL-c level. Rice wine decreased both TG level and blood glucose in women only and it could be one of healthy alcoholic beverages in MS prevention in Chinese women. While excessive liquor consumption increased BP and waist circumference level and it may lead to hypertension and central obesity in Chinese men.

Keywords: Metabolic syndrome, Components, Alcohol consumption, Rice wine, Liquor

\footnotetext{
* Correspondence: huicai2012@yahoo.com

'Department of Epidemiology and Medical Statistics, School of Public Health,

Nantong University, Nantong, Jiangsu, China

Full list of author information is available at the end of the article
} 


\section{Introduction}

Metabolic syndrome (MS) is a constellation of metabolic abnormalities, including abdominal obesity, hypertriglyceridemia, low high-density lipoprotein (HDL) concentrations, hypertension, and hyperglycaemia [1], which is strong association with the development of type II diabetes and risk of cardiovascular morbidity and mortality $[2,3]$. Ford $e t$ al. reported that according to International Diabetes Federation (IDF) criteria the prevalence of MS is $23 \%$ in America, and $23.50 \%$ and $14.70 \%$ in urban and rural areas in China, respectively [4]. Prevalence and incidence of MS increase rapidly and become a major public health challenge worldwide [5]. The etiology of the metabolic syndrome is complex, determined by the interplay of both genetic and environmental factors [6]. There is increasing interest to obtain a greater understanding of the modifiable factors that may mitigate or moderate the progression of MS leading towards its development.

Alcohol consumption is one of most prevalent lifestyles in the world [7]. It has recently been interested in its association with risk of MS and its components. Several studies reported that alcohol consumption has a higher risk of developing type II diabetes [8,9], hypertension $[8,10-12]$, obesity [8,13-15], high triglycerides (TG) $[10,16,17]$ and high fasting glucose [1], which are the principal components of MS [8]. Sayon et al. found that severe drinkers were associated with weight gain while light to moderate alcohol drinkers, especially wine drinkers, was not associated with obesity [18]. However, many studies have suggested that alcohol consumption is a protective effect on MS prevalence and incidence by increasing HDL cholesterol (HDL-c) $[10,19,20]$. Stoutenberg et al. reported that all levels of alcohol consumption provided significant inverse associations with MS but alcohol consumption was not significantly associated with central obesity, hypertriacylglycerolemia or hypertension [21]. Several studies have further reported that moderate alcohol consumption was associated with a lower risk of diabetes [22-24]. Take these inconsistent results into consideration, it is unclear about association between different alcoholic beverages and MS components. Recently, several studies conducted in China have evaluated association between alcohol consumption and MS among Chinese minors [11] and Chinese men [1]. Few studies focus on evaluating the associations between the amount and types of alcoholic beverage consumed and MS components among Chinese adults.

We launched the Nantong Metabolic Syndrome Study (NMSS) in 2007. The NMSS is a population-based cross-sectional study of 20,502 participants $(6,997$ men and 13,505 women) aged 18-74 years in the rural areas of Nantong, China. In current study we will evaluate association between several alcoholic beverages and MS components in both men and women living in rural
China and to our knowledge, this is the largest study on association between alcohol consumption and each of MS components in rural China.

\section{Methods \\ Study population}

The NMSS recruitment began in July 2007 and was completed in August 2008. The details of the NMSS have been described elsewhere [25]. Briefly, we recruited 24,519 residents between the ages of 18 and 74 years from two townships in rural Nantong. Among them, 20,502 participants, including 6,997 men and 13,505 women, were enrolled in the study, with a response rate of $83.6 \%$. The reasons for non-participation were refusal (3.21\%), out of the area during enrollment (7.21\%), and other miscellaneous reasons, such as poor health or hearing problems (5.98\%). In our study, a rural area was defined as an area with a primary administrative unit termed a 'village'. At the time of interview, most participants (99.5\%) lived in a village, and 13,306 (64.9\%) people were farmers. The study protocols were approved by the Boards of Scientific Research of Nantong University and the Nantong Centers for Disease Control (CDC), and all participants provided written informed consent. Socioeconomic factors, dietary intake, physical activity, alcohol consumption, smoking status, personal medical history and family history of several chronic diseases were assessed using the same standard questionnaires. The height, weight, and waist circumference of each participant was measured according to standard protocols.

The participants were asked the age they started and stopped smoking and how many cigarettes they consumed per day. We defined ever smokers as participants who had smoked at least 100 cigarettes in their lifetimes. Each participant were asked about monthly alcohol consumption within the recent year, they provided the usual alcohol amount of grape wine, rice wine, beer and liquor consumed on a monthly basis separately. One drink was defined as 4 ounces of grape wine, 4.8 ounces of rice wine, a 12 ounce of beer or 1 ounce of liquor, all of which contain approximately 0.5 ounces of absolute alcohol [26]. A total of 25.6\% (11.2\% female and 53.6\% male) of the participants in our study consumed alcohol, $66.2 \%$ (66.6\% male and $65.1 \%$ female respectively) of whom reportedly consumed rice wine; $1.8 \%$ was grape wine drinkers. Majority of people in rural China consumes rice wine rather than grape wine, so we combined them together to form a rice wine consumption group. Participants who consumed any kinds of alcoholic beverage at least 12 times last year were defined as a drinker in current study. All drinkers were classified into four categories based on their alcohol intake from, for example, all sources of alcoholic intake: non-drinkers, light drinkers ( $\leq 5.7$ grams/day for women and $\leq 16.4$ grams/ 
day for men), moderate drinkers ( $\leq 17.7$ grams/day for women and $\leq 45.2$ grams/day for men), and severe drinkers ( $>17.7$ grams/day for women and $>45.2$ grams/ day for men). We investigated the intake amount of some foods in liang (=50 grams), including red meat (e.g., pork, beef, and lamb), white meat (e.g., chicken, duck, and goose), and fish. The data of other foods consumed, such as vegetables, fruits, and soy foods were also collected. We asked the participants how frequently (daily, weekly, monthly, yearly, or never) they consumed these food groups over the past year, followed by a question on the amount consumed in liang per unit of time. Exercise was defined as the participants who performed physical activities, such as Qigong, jogging, and basketball, during their leisure time. Tea consumption was defined as drinking tea more than two times per week for at least six months continuously. Socio-demographic factors, such as age at interview, education (none, elementary school, middle/ high school, college, and above), personal income in Yuan/month ( $\leq 500,501-1000, \geq 1001)$ and occupation, were analyzed in the study as potential confounders.

\section{Anthropometric and biochemical measurements}

According to standard protocol, anthropometric measurements of weight, height and waist circumference were taken twice for each participant during the inperson interviewers, which might help to prevent reading and typing errors. A third measurement was taken if difference between the first two measurements was larger than $1 \mathrm{~cm}$ for height and waist circumference or 1 kilogram $(\mathrm{kg})$ for weight. The intra-observer variations of the three anthropometric measures were small, and the average coefficients of variation were $0.27 \%, 0.02 \%$, and $0.24 \%$ for height, weight and waist circumference, respectively. Therefore, the average of two readings of height, weight, and waist circumference was used in our study. Based on the anthropometric measures, body mass index (BMI) was calculated as weight in kilograms divided by the square of height in meters.

Systolic blood pressure (SBP) and diastolic blood pressure (DBP) were measured three times with a standardized mercury sphygmomanometer after the participants had rested for five minutes or longer in a sitting position. The mean of the three measurements was used in the analysis.

To measure serum lipids and glucose, a $10 \mathrm{~mL}$-overnight fasting blood sample was drawn into an EDTA vacutainer tube at the time of the in-person interview. The fasting time was verified before the blood sample collection, and the participants who had not fasted for at least eight hours did not have their blood drawn. The samples were stored in a portable Styrofoam box with ice packs $\left(0-4^{\circ} \mathrm{C}\right)$ and were sent to a central CDC laboratory in Nantong. Serum samples were obtained by centrifugation. Glucose and lipid levels were measured within six hours of the sample separation. We stored the remaining specimens at $-70^{\circ} \mathrm{C}$ to conduct other laboratory assays. Serum glucose, HDL cholesterol and triglyceride levels were analyzed enzymatically using reagents from the Shino-Test Corporation, Japan. An automated chemistry analyzer (Hitachi 7180, Tokyo, Japan) was used to measure the serum levels of glucose and the lipid profiles of the 20,502 participants in the Nantong CDC. Both inter- and intra- assay variations (coefficient of variation, $\mathrm{CV}$ ) were less than $3.5 \%$ for glucose, TG, and HDL-c.

\section{Criteria for the MS diagnosis}

Criteria of metabolic syndrome diagnosis according to a joint interim statement of the International Diabetes Federation Task Force on Epidemiology and Prevention; National Heart, Lung, and Blood Institute; American Heart Association; World Heart Federation; International Atherosclerosis Society; and International Association for the Study of Obesity [27] by adopting the Asian criteria for waist circumference. MS was defined as the presence of any 3 or more of the following 5 metabolic risk factors: (1) central obesity (waist circumference $\geq 80 \mathrm{~cm}$ for Chinese women and $\geq 85 \mathrm{~cm}$ for Chinese men); (2) elevated TG (fasting serum TG $\geq$ $1.7 \mathrm{mmol} / \mathrm{L}$ or taking abnormal lipid medication); (3) reduced HDL-c (fasting serum HDL-c $<1.3 \mathrm{mmol} / \mathrm{L}$ for Chinese women and HDL-c $<1.0 \mathrm{mmol} / \mathrm{L}$ for Chinese men or specific treatment for this lipid abnormality); (4) elevated blood pressure (SBP $\geq 130 \mathrm{mmHg}$ or $\mathrm{DBP} \geq 85 \mathrm{mmHg}$ or taking hypertension medication); (5) elevated fasting glucose (serum glucose level $\geq 6.0 \mathrm{mmol} / \mathrm{L}$ or taking diabetes medication).

\section{Statistical analyses}

Demographic, dietary and lifestyle characteristics were described as the mean \pm standard deviation (SD) (or Median \pm IQR) for continuous variables and percentages for categorical variables. These parameters were compared between the drinkers and non-drinkers using ANOVA for continuous variables with normal distribution, Wilcoxon rank sum test for continuous variables with non-normal distribution, and chis-square test for categorical variables. ANOVA was also applied to compare MS components between any type of alcoholic beverages drinkers and non-drinkers. Odds Ratios (ORs) and 95\% confidence intervals (CIs) were estimated using non-conditional logistic regression to assess the associations between alcohol consumption and MS components. The regression model was adjusted for age at interview, BMI, education, marriage status, personal income, occupation, exercise, smoking status, tea consumption, and the intake of meat, fish, soy food, fruits and vegetables. The test for linear trend was 
performed by entering the ordinal exposure (e.g., the amount of alcohol from beer, liquor, rice wine and all sources) as continuous parameters in the models. A restricted cubic spline model was used for curve fitting between the ORs of MS components (TG, HDL-c, glucose, SBP and DBP, waist circumference) and alcohol consumption (continuous variable), and the figures present the curve fitting results. All $\mathrm{p}$ values presented were based on two-tailed test, and $\mathrm{p}<0.05$ was considered statistically significant. All analyses were performed using SAS statistical software (version 9.2; SAS Institute, Cary, NC).

\section{Results}

Characteristics of study participants

Differences of selected demographic characteristics, lifestyle factors, anthropometric measurements and some food intakes between drinkers and non-drinkers are reported in Table 1. There were 5,253 (25.6\%) drinkers

Table 1 Characteristics of the study population by gender and drinking status*

\begin{tabular}{|c|c|c|c|c|c|c|c|}
\hline & \multirow[t]{2}{*}{ All participants } & \multicolumn{2}{|l|}{ Women } & \multirow[b]{2}{*}{$p$ values } & \multicolumn{2}{|l|}{ Men } & \multirow[b]{2}{*}{$p$ values } \\
\hline & & $\begin{array}{l}\text { Drinker } \\
(n=1,506)\end{array}$ & $\begin{array}{l}\text { Non-drinker } \\
(n=11,999)\end{array}$ & & $\begin{array}{l}\text { Drinker } \\
(\mathrm{n}=3747)\end{array}$ & $\begin{array}{l}\text { Non-drinker } \\
(\mathrm{n}=3250)\end{array}$ & \\
\hline Age at interview (yeas, median $\pm I Q R^{\dagger}$ ) & $55.7 \pm 18.2$ & $57.8 \pm 14.2$ & $53.8 \pm 17.7$ & $<0.001$ & $57.6 \pm 15.3$ & $59.5 \pm 16.9$ & $<0.001$ \\
\hline Weight (kg, mean \pm SD) & $60.2 \pm 0.1$ & $58.1 \pm 0.3$ & $58.2 \pm 0.1$ & 0.855 & $64.7 \pm 0.2$ & $63.6 \pm 0.2$ & $<0.001$ \\
\hline $\mathrm{BMI}\left(\mathrm{kg} / \mathrm{m}^{2}\right.$, mean $\left.\pm \mathrm{SD}\right)$ & $23.7 \pm 0.0$ & $23.7 \pm 0.1$ & $24.0 \pm 0.0$ & 0.029 & $23.3 \pm 0.1$ & $23.2 \pm 0.1$ & 0.020 \\
\hline Red meat (g/day, median \pm IQR) & $18.9 \pm 22.8$ & $18.9 \pm 24.2$ & $18.9 \pm 24.2$ & 0.169 & $25.1 \pm 25.1$ & $18.9 \pm 18.9$ & $<0.001$ \\
\hline White meat (g/day, median $\pm I Q R)$ & $10.8 \pm 18.2$ & $10.8 \pm 17.0$ & $10.8 \pm 17.2$ & 0.473 & $14.1 \pm 18.2$ & $10.8 \pm 18.2$ & $<0.001$ \\
\hline Fish (g/day, median \pm IQR) & $22.1 \pm 34.1$ & $20.4 \pm 34.1$ & $20.4 \pm 26.6$ & 0.159 & $22.1 \pm 31.0$ & $22.1 \pm 35.4$ & $<0.001$ \\
\hline Vegetables (g/day, median \pm IQR) & $225.0 \pm 187.5$ & $267.9 \pm 187.5$ & $225.0 \pm 225.0$ & 0.013 & $300.0 \pm 187.5$ & $262.5 \pm 225.0$ & 0.017 \\
\hline Fruits (g/day, median \pm IQR) & $17.5 \pm 41.5$ & $13.7 \pm 41.5$ & $17.5 \pm 41.5$ & 0.003 & $18.0 \pm 41.3$ & $18.3 \pm 41.3$ & 0.626 \\
\hline Soy food (g/day, median \pm IQR) & $28.6 \pm 57.1$ & $28.0 \pm 35.7$ & $28.6 \pm 57.1$ & $<0.001$ & $32.9 \pm 57.1$ & $32.9 \pm 57.1$ & 0.450 \\
\hline MS (\%) & 21.1 & 20.6 & 23.6 & 0.009 & 16.4 & 17.1 & 0.477 \\
\hline \multicolumn{8}{|l|}{ Education (\%) } \\
\hline Primary school/under & 59.7 & 69.1 & 63.3 & & 50.8 & 52.8 & \\
\hline Middle school & 27.9 & 22.0 & 26.9 & & 31.1 & 30.1 & \\
\hline High school/above & 12.4 & 8.9 & 9.8 & $<0.001$ & 18.1 & 17.1 & 0.057 \\
\hline \multicolumn{8}{|l|}{ Marriage (\%) } \\
\hline Yes & 90.0 & 90.7 & 90.1 & & 91.1 & 88.2 & \\
\hline $\mathrm{No}^{\#}$ & 10.0 & 9.3 & 9.9 & 0.644 & 9.0 & 11.8 & $<0.001$ \\
\hline \multicolumn{8}{|l|}{ Income per person (\%) } \\
\hline$\leq 500$ Yuan & 64.3 & 63.0 & 65.2 & & 61.4 & 64.4 & \\
\hline 501-1000 Yuan & 29.1 & 29.6 & 29.2 & & 29.8 & 28.0 & \\
\hline$\geq 1001$ Yuan & 6.6 & 7.4 & 5.6 & 0.294 & 8.8 & 7.7 & 0.007 \\
\hline \multicolumn{8}{|l|}{ Farmer (\%) } \\
\hline Yes & 64.9 & 73.5 & 68.6 & & 55.3 & 59.0 & \\
\hline No & 35.1 & 26.5 & 31.4 & $<0.001$ & 44.7 & 41.0 & $<0.001$ \\
\hline \multicolumn{8}{|l|}{ Ever smoked (\%) } \\
\hline Yes & 18.8 & 11.5 & 2.7 & & 58.3 & 35.4 & \\
\hline No & 81.2 & 88.5 & 97.3 & $<0.001$ & 41.7 & 64.6 & $<0.001$ \\
\hline \multicolumn{8}{|l|}{ Tea consumption (\%) } \\
\hline Yes & 15.2 & 15.1 & 10.0 & & 28.4 & 19.3 & \\
\hline No & 84.8 & 84.9 & 90.0 & $<0.001$ & 71.6 & 80.7 & $<0.001$ \\
\hline \multicolumn{8}{|l|}{ Exercise (\%) } \\
\hline Yes & 9.3 & 8.8 & 10.1 & & 8.2 & 7.7 & \\
\hline No & 90.7 & 91.2 & 89.9 & 0.092 & 91.8 & 92.3 & 0.508 \\
\hline
\end{tabular}


including 1,506 (11.2\%) female and 3,747 (53.6\%) male in our study. The MS prevalence was $20.6 \%$ for drinkers, which was 3.0\% lower than that for nondrinkers $(P=0.009)$ in women. But the prevalence of MS was comparable between drinkers and nondrinkers in men. Drinkers were more likely to be smokers and tea consumers, to have healthy vegetables among all participants. Female drinkers were older and most of them were farmers. They were less educated and less likely to be obesity and to take fruit and soy food, compared with female non-drinkers. But male drinkers were younger and had higher income, more likely to be obesity and to take meat and less likely to be farmers.

\section{Association of alcohol consumption and MS components}

We found different levels of MS components between drinking status and three types of alcoholic beverages after adjusted for age at interview in Table 2. In general, drinkers, no matter what kind of alcoholic beverage they drunk, had a higher level of HDL-c, compared with non-drinkers. Female drinkers had a lower level of TG $(\mathrm{P}<0.01)$, and SBP $(\mathrm{P}<0.05)$, compared with nondrinkers. Furthermore, lower SBP were found among female participants who consumed beer, liquor or rice wine. But only lower level of TG was found in rice wine drinkers, compared with non-drinkers. There was no difference of waist circumference, glucose level, and DBP between female drinkers and non-drinkers. Differently, higher levels of waist circumference, TG, SBP, and DBP (all $\mathrm{P}<0.01$ ) were found in male drinkers, compared with non-drinkers. Also, higher levels of waist circumference, TG and DBP were detected in beer or liquor drinkers. While higher glucose and SBP were only found in liquor drinkers, compared with non-drinkers.

Table 3 shows the association of MS components with consumption of several alcoholic beverages adjusted for age at interview, BMI, education, marriage status, personal income, occupation, exercise, smoking status, tea consumption and intake of meat, fish, soy food, fruits and vegetables among female participants. We found that rice wine consumption was associated with decreasing risk of high TG $(\mathrm{OR}=0.88,95 \%$ CI: 0.81-0.95), risk of high glucose $(\mathrm{OR}=0.79,95 \% \mathrm{CI}: 0.67-0.93)$ and risk of low HDL-c (OR $=0.84,95 \%$ CI: 0.77-0.91). There was no association between rice wine consumption and blood pressure or waist circumference. However, beer consumption was only negatively associated with high risk of TG (OR $=0.87,95 \% \mathrm{CI}$ : $0.80-0.95)$ and risk of low HDL-c (OR $=0.82$, 95\% CI: 0.75-0.90).

The associations of MS components with consumption of several alcoholic beverages among male participants are presented in Table 4. Similar to that among women, a decreased risk of having low HDL-c was found in rice wine ( $\mathrm{OR}=0.75,95 \% \mathrm{CI}$ : $0.66-0.87$ ), beer $(\mathrm{OR}=0.74,95 \% \mathrm{CI}: 0.65-0.85)$, and liquor drinkers (OR $=0.82$, 95\% CI: 0.71-0.94), compared with nondrinkers. Moreover, liquor consumption was associated with increasing risk of high SBP $(\mathrm{OR}=1.08,95 \%$ CI: 1.03-1.15), high DBP (OR $=1.15,95 \% \mathrm{CI}: 1.08$ $1.23)$, and high waist circumference $(\mathrm{OR}=1.11,95 \%$ CI: 1.02-1.20). There was no association between liquor consumption and TG or glucose level.

Associations of all alcoholic beverage and rice wine in women or liquor in men with MS components are depicted in Figure 1. In women we found that both all alcoholic beverage and rice wine had strong protective effect on high TG, high blood glucose and low HDL when consumption of alcohol was less than 10 grams per day. The protective effect of rice wine was increased a little bit on high glucose and low HDL if rice wine consumption was more than 10 grams per day. In contrast, both all alcoholic beverage and liquor were significantly associated with the prevalence of high BP in men. Also, a similar association was found between liquor and the prevalence of central obesity.

\section{Discussion}

In this cross-sectional study among adults in rural China, alcohol consumption was associated with a lower prevalence of MS in women and any alcoholic beverage might decrease the risk of low HDL-c in both men and women. In particular, we observed that both rice wine and light to moderate alcoholic beverage consumption had a significantly protective effect on high TG, and high glucose with a non-linear relationship among women, but not among men. However, severe liquor and alcoholic beverage consumption was found to increase risk of high BP and central obesity among men only.

Reports on the relationship between alcohol consumption and MS were inconsistent. Some studies have shown a positive, linear relationship between alcohol consumption and MS $[1,28,29]$ and this association was significantly higher in men aged 50 years or older, whereas no significant association was observed in women [30]. Other studies reported that mild-tomoderate alcohol consumption decreased the risk of the MS in all participants [31] or in women only [32]. In our previous study, we found that rice wine consumption may decrease the risk of the MS in women and no associations were found in beer or liquor consumption [25]. These inconsistent results might be related to the different type and amount of alcoholic beverage consumption, the complex mechanistic relationship between alcohol consumption and each component of MS, and some confounding factors such as race and sex $[28,29,33]$. 
Table 2 Comparison of the levels of MS components according to drinking status and the three types of alcoholic beverages* (mean \pm SD)

\begin{tabular}{|c|c|c|c|c|c|c|c|c|c|c|c|c|}
\hline & \multicolumn{3}{|l|}{ Drinker } & \multicolumn{3}{|l|}{ Beer drinker } & \multicolumn{3}{|c|}{ Liquor drinker } & \multicolumn{3}{|c|}{ Rice wine drinker } \\
\hline & Yes & No & $p$ & Yes & No & $p$ & Yes & No & $p$ & Yes & No & $p$ \\
\hline & \multicolumn{12}{|l|}{ For women } \\
\hline Waist (cm) & $81.0 \pm 0.26$ & $80.9 \pm 0.09$ & 0.88 & $81.4 \pm 0.31$ & $80.9 \pm 0.09$ & 0.13 & $81.5 \pm 0.48$ & $80.9 \pm 0.09$ & 0.22 & $80.8 \pm 0.33$ & $81.0 \pm 0.09$ & 0.58 \\
\hline TG (mmol/L) & $1.35 \pm 0.03$ & $1.46 \pm 0.01$ & $<0.01$ & $1.39 \pm 0.04$ & $1.46 \pm 0.01$ & 0.07 & $1.44 \pm 0.06$ & $1.45 \pm 0.01$ & 0.92 & $1.36 \pm 0.04$ & $1.46 \pm 0.01$ & 0.02 \\
\hline HDL-c (mmol/L) & $1.66 \pm 0.01$ & $1.56 \pm 0.01$ & $<0.01$ & $1.66 \pm 0.01$ & $1.56 \pm 0.01$ & $<0.01$ & $1.65 \pm 0.02$ & $1.57 \pm 0.01$ & $<0.01$ & $1.66 \pm 0.01$ & $1.56 \pm 0.01$ & $<0.01$ \\
\hline Glucose (mmol/L) & $4.48 \pm 0.04$ & $4.54 \pm 0.01$ & 0.10 & $4.51 \pm 0.04$ & $4.54 \pm 0.01$ & 0.57 & $4.48 \pm 0.07$ & $4.54 \pm 0.01$ & 0.39 & $4.45 \pm 0.05$ & $4.54 \pm 0.01$ & 0.06 \\
\hline SBP (mmHg) & $120.3 \pm 0.46$ & $121.4 \pm 0.16$ & 0.02 & $120.3 \pm 0.54$ & $121.4 \pm 0.16$ & 0.05 & $119.6 \pm 0.84$ & $121.3 \pm 0.15$ & 0.05 & $119.6 \pm 0.56$ & $121.4 \pm 0.16$ & $<0.01$ \\
\hline \multirow[t]{2}{*}{$\mathrm{DBP}(\mathrm{mmHg})$} & $73.3 \pm 0.28$ & $73.5 \pm 0.10$ & 0.63 & $73.5 \pm 0.33$ & $73.4 \pm 0.10$ & 0.84 & $73.3 \pm 0.51$ & $73.5 \pm 0.09$ & 0.81 & $72.9 \pm 0.34$ & $73.5 \pm 0.09$ & 0.12 \\
\hline & \multicolumn{12}{|l|}{ For men } \\
\hline Waist (cm) & $83.3 \pm 0.17$ & $82.1 \pm 0.18$ & $<0.01$ & $83.5 \pm 0.20$ & $82.3 \pm 0.16$ & $<0.01$ & $84.1 \pm 0.24$ & $82.3 \pm 0.14$ & $<0.01$ & $82.9 \pm 0.21$ & $82.7 \pm 0.15$ & 0.58 \\
\hline TG (mmol/L) & $1.49 \pm 0.02$ & $1.35 \pm 0.03$ & $<0.01$ & $1.50 \pm 0.03$ & $1.38 \pm 0.02$ & $<0.01$ & $1.58 \pm 0.03$ & $1.37 \pm 0.02$ & $<0.01$ & $1.45 \pm 0.03$ & $1.41 \pm 0.02$ & 0.35 \\
\hline $\mathrm{HDL-c}(\mathrm{mmol} / \mathrm{L})$ & $1.62 \pm 0.01$ & $1.48 \pm 0.01$ & $<0.01$ & $1.62 \pm 0.01$ & $1.52 \pm 0.01$ & $<0.01$ & $1.63 \pm 0.01$ & $1.53 \pm 0.01$ & $<0.01$ & $1.64 \pm 0.01$ & $1.51 \pm 0.01$ & $<0.01$ \\
\hline Glucose (mmol/L) & $4.51 \pm 0.03$ & $4.45 \pm 0.03$ & 0.13 & $4.51 \pm 0.03$ & $4.47 \pm 0.03$ & 0.29 & $4.59 \pm 0.04$ & $4.45 \pm 0.02$ & $<0.01$ & $4.46 \pm 0.03$ & $4.50 \pm 0.02$ & 0.40 \\
\hline SBP (mmHg) & $126.2 \pm 0.28$ & $124.3 \pm 0.30$ & $<0.01$ & $125.6 \pm 0.34$ & $125.2 \pm 0.26$ & 0.29 & $126.9 \pm 0.40$ & $124.8 \pm 0.24$ & $<0.01$ & $125.5 \pm 0.34$ & $125.3 \pm 0.26$ & 0.63 \\
\hline DBP $(\mathrm{mmHg})$ & $76.4 \pm 0.18$ & $74.8 \pm 0.19$ & $<0.01$ & $76.1 \pm 0.21$ & $75.3 \pm 0.17$ & $<0.01$ & $77.1 \pm 0.25$ & $75.1 \pm 0.15$ & $<0.01$ & $76.0 \pm 0.22$ & $75.4 \pm 0.16$ & 0.02 \\
\hline
\end{tabular}


Table 3 Associations of MS components with consumption of several alcoholic beverages among cases of high MS components and healthy women*

\begin{tabular}{|c|c|c|c|c|c|c|c|c|c|c|c|c|}
\hline & \multicolumn{2}{|l|}{ TG } & \multicolumn{2}{|l|}{ HDL } & \multicolumn{2}{|l|}{ Glucose } & \multicolumn{2}{|l|}{ SBP } & \multicolumn{2}{|l|}{ DBP } & \multicolumn{2}{|c|}{ Waist circumference } \\
\hline & Cases (\%) & OR $(95 \% \mathrm{Cl})$ & Cases (\%) & OR $(95 \% \mathrm{Cl})$ & Cases (\%) & OR $(95 \% \mathrm{Cl})$ & Cases (\%) & OR $(95 \% \mathrm{Cl})$ & Cases (\%) & OR $(95 \% \mathrm{Cl})$ & Cases (\%) & OR $(95 \% \mathrm{Cl})$ \\
\hline \multicolumn{13}{|c|}{ Alcohol from beer (g/d) } \\
\hline 0 & $3078(26.6)$ & 1.0 & $3048(27.4)$ & 1.0 & $610(6.1)$ & 1.0 & $3414(29.9)$ & 1.0 & 1757(16.6) & 1.0 & 6499(53.0) & 1.0 \\
\hline$\leq 3.1$ & $102(24.5)$ & $0.78(0.61-1.00)$ & 83(21.0) & $0.69(0.54-0.89)$ & $14(3.9)$ & $0.57(0.33-0.98)$ & 123(29.9) & $0.86(0.68-1.09)$ & $71(18.3)$ & $1.04(0.79-1.38)$ & $257(59.0)$ & $1.12(0.85-1.48)$ \\
\hline$\leq 6.2$ & $86(23.4)$ & $0.76(0.58-0.99)$ & 73(20.4) & $0.70(0.53-0.91)$ & $22(6.7)$ & $0.94(0.59-1.48)$ & $121(32.3)$ & $0.99(0.78-1.25)$ & $64(18.5)$ & $1.16(0.86-1.55)$ & $217(54.5)$ & $1.00(0.74-1.34)$ \\
\hline$>6.2$ & $42(22.1)$ & $0.72(0.50-1.04)$ & 36(18.9) & $0.61(0.42-0.88)$ & $7(4.1)$ & $0.56(0.26-1.22)$ & $56(29.6)$ & $0.81(0.57-1.15)$ & 28(15.6) & $0.85(0.55-1.32)$ & $117(57.6)$ & $1.30(0.88-1.92)$ \\
\hline$p$ for trend & & 0.0018 & & $<0.0001$ & & 0.0723 & & 0.2106 & & 0.8447 & & 0.2457 \\
\hline \multicolumn{13}{|c|}{ Alcohol from liquor (g/d) } \\
\hline 0 & $3206(26.4)$ & 1.0 & $3158(27.0)$ & 1.0 & $632(6.0)$ & 1.0 & 3592(29.9) & 1.0 & 1866(16.8) & 1.0 & 6859(53.2) & 1.0 \\
\hline$\leq 6.2$ & $41(24.6)$ & $0.75(0.52-1.10)$ & 39(24.8) & $0.87(0.60-1.28)$ & $11(7.6)$ & $1.22(0.64-2.31)$ & $55(33.3)$ & $1.05(0.73-1.50)$ & $25(17.1)$ & $1.00(0.63-1.58)$ & $95(54.0)$ & $0.73(0.47-1.13)$ \\
\hline$\leq 20.5$ & $26(24.8)$ & $0.71(0.44-1.14)$ & $17(17.4)$ & $0.53(0.31-0.92)$ & $2(2.3)$ & $0.29(0.07-1.18)$ & $25(25.8)$ & $0.64(0.39-1.04)$ & $9(9.7)$ & $0.47(0.23-0.95)$ & 62(57.9) & $0.91(0.50-1.64)$ \\
\hline$>20.5$ & $38(28.2)$ & $1.00(0.66-1.49)$ & $27(20.5)$ & $0.70(0.45-1.09)$ & $10(8.3)$ & $1.11(0.56-2.20)$ & 44(32.4) & $0.88(0.59-1.31)$ & $20(16.3)$ & $0.90(0.54-1.51)$ & $82(56.2)$ & $1.15(0.71-1.87)$ \\
\hline$p$ for trend & & 0.2644 & & 0.0105 & & 0.6687 & & 0.1981 & & 0.1830 & & 0.9317 \\
\hline \multicolumn{13}{|c|}{ Alcohol from rice wine $(\mathrm{g} / \mathrm{d})$} \\
\hline 0 & $3093(26.7)$ & 1.0 & $3070(27.4)$ & 1.0 & $618(6.1)$ & 1.0 & $3419(29.8)$ & 1.0 & 1788(16.8) & 1.0 & $6561(53.1)$ & 1.0 \\
\hline$\leq 4.1$ & $68(22.4)$ & $0.65(0.49-0.87)$ & $60(20.6)$ & $0.66(0.49-0.88)$ & $12(4.5)$ & $0.61(0.33-1.10)$ & $90(29.7)$ & $0.82(0.63-1.08)$ & $42(15.1)$ & $0.79(0.55-1.12)$ & 183(56.8) & $0.94(0.68-1.30)$ \\
\hline$\leq 12.8$ & 73(24.6) & $0.74(0.56-0.99)$ & $50(17.9)$ & $0.60(0.43-0.82)$ & $12(4.6)$ & $0.57(0.31-0.98)$ & $91(31.5)$ & $0.79(0.60-1.04)$ & $46(17.0)$ & $0.96(0.68-1.35)$ & $174(57.1)$ & $1.05(0.75-1.46)$ \\
\hline$>12.8$ & $78(23.7)$ & $0.76(0.58-0.98)$ & 63(19.8) & $0.70(0.53-0.94)$ & $13(4.5)$ & $0.57(0.32-0.99)$ & $117(36.7)$ & $1.10(0.85-1.42)$ & $43(14.8)$ & $0.87(0.62-1.23)$ & 181(53.9) & $1.17(0.85-1.61)$ \\
\hline$p$ for trend & & 0.0013 & & $<0.0001$ & & 0.0044 & & 0.5820 & & 0.2929 & & 0.4059 \\
\hline \multicolumn{13}{|c|}{ All sources of alcohol $(\mathrm{g} / \mathrm{d})$} \\
\hline 0 & $2982(26.8)$ & 1.0 & $2972(27.7)$ & 1.0 & $593(6.2)$ & 1.0 & $3265(29.7)$ & 1.0 & $1701(16.7)$ & 1.0 & $6262(52.9)$ & 1.0 \\
\hline$\leq 5.7$ & 109(23.4) & $0.72(0.57-0.91)$ & $87(19.7)$ & $0.62(0.49-0.80)$ & $16(4.0)$ & $0.52(0.31-0.87)$ & 138(29.9) & $0.85(0.68-1.07)$ & $81(18.5)$ & $1.06(0.81-1.38)$ & $278(56.1)$ & $0.99(0.76-1.29)$ \\
\hline$\leq 17.7$ & $111(23.6)$ & $0.72(0.57-0.90)$ & $105(22.9)$ & $0.78(0.62-0.98)$ & $18(4.4)$ & $0.56(0.35-0.92)$ & $151(32.1)$ & $0.90(0.73-1.12)$ & $66(15.4)$ & $0.84(0.64-1.21)$ & 285(57.9) & $1.20(0.92-1.55)$ \\
\hline$>17.7$ & $110(23.4)$ & $0.73(0.57-0.92)$ & 79(17.4) & $0.57(0.44-0.74)$ & $27(6.4)$ & $0.81(0.53-1.23)$ & 162(35.1) & $1.00(0.81-1.25)$ & $72(16.7)$ & $0.99(0.75-1.30)$ & $274(55.7)$ & $1.19(0.91-1.55)$ \\
\hline$p$ for trend & & $<0.0001$ & & $<0.0001$ & & 0.0144 & & 0.4517 & & 0.5634 & & 0.1018 \\
\hline
\end{tabular}

*Abnormal TG, HDL, Glucose and blood pressure, respectively. All models were adjusted for age at interview, BMI, education, marriage status, personal income, occupation, exercise, smoking status, tea consumption, and intake of meat, fish, soy food, fruits and vegetables. 
Table 4 Associations of MS components with consumption of several alcoholic beverages among cases of high MS components and healthy men*

\begin{tabular}{|c|c|c|c|c|c|c|c|c|c|c|c|c|}
\hline & \multicolumn{2}{|l|}{ TG } & \multicolumn{2}{|l|}{ HDL } & \multicolumn{2}{|l|}{ Glucose } & \multicolumn{2}{|l|}{ SBP } & \multicolumn{2}{|l|}{ DBP } & \multicolumn{2}{|c|}{ Waist circumference } \\
\hline & Cases (\%) & OR $(95 \% \mathrm{Cl})$ & Cases (\%) & OR $(95 \% \mathrm{Cl})$ & Cases (\%) & OR $(95 \% \mathrm{Cl})$ & Cases (\%) & OR $(95 \% \mathrm{Cl})$ & Cases (\%) & OR $(95 \% \mathrm{Cl})$ & Cases (\%) & OR $(95 \% \mathrm{Cl})$ \\
\hline \multicolumn{13}{|c|}{ Alcohol from beer (g/d) } \\
\hline 0 & $873(21.2)$ & 1.0 & $241(6.4)$ & 1.0 & $222(5.9)$ & 1.0 & 1457(34.9) & 1.0 & $804(20.3)$ & 1.0 & 1668(38.8) & 1.0 \\
\hline$\leq 6.2$ & $314(22.6)$ & $0.94(0.79-1.10)$ & $48(3.8)$ & $0.49(0.35-0.68)$ & $70(5.4)$ & $0.81(0.61-1.09)$ & $485(34.3)$ & $1.00(0.87-1.14)$ & $273(20.0)$ & $0.92(0.78-1.09)$ & $630(43.5)$ & $1.15(0.95-1.39)$ \\
\hline$\leq 12.3$ & $71(27.7)$ & $1.11(0.80-1.52)$ & $9(3.9)$ & $0.41(0.20-0.84)$ & $10(4.4)$ & $0.66(0.34-1.29)$ & $93(35.8)$ & $1.08(0.82-1.43)$ & $53(21.8)$ & $1.00(0.71-1.39)$ & $127(46.9)$ & $1.29(0.89-1.87)$ \\
\hline$>12.3$ & $230(27.4)$ & $1.15(0.95-1.39)$ & $32(4.3)$ & $0.51(0.34-0.75)$ & $34(4.5)$ & $0.70(0.47-1.03)$ & 290(34.4) & $1.07(0.91-1.27)$ & $177(21.8)$ & $1.03(0.84-1.26)$ & $374(43.1)$ & $1.07(0.85-1.36)$ \\
\hline$p$ for trend & & 0.1967 & & $<0.0001$ & & 0.0308 & & 0.3972 & & 0.9232 & & 0.2986 \\
\hline \multicolumn{13}{|c|}{ Alcohol from liquor (g/d) } \\
\hline 0 & $1049(21.4)$ & 1.0 & $258(5.8)$ & 1.0 & $240(5.4)$ & 1.0 & 1675(33.7) & 1.0 & 893(18.9) & 1.0 & 1982(38.8) & 1.0 \\
\hline$\leq 12.3$ & $130(25.0)$ & $1.01(0.80-1.28)$ & $21(4.5)$ & $0.60(0.37-0.97)$ & $30(6.3)$ & $0.97(0.64-1.47)$ & 174(33.7) & $0.99(0.80-1.22)$ & 103(20.6) & $1.03(0.81-1.32)$ & $245(45.4)$ & 1.32(0.99-1.76) \\
\hline$\leq 37.0$ & 180(26.6) & $1.16(0.94-1.43)$ & $32(5.2)$ & $0.72(0.48-1.07)$ & $35(5.7)$ & $0.96(0.66-1.41)$ & 256(36.9) & $1.14(0.95-1.36)$ & $164(24.7)$ & $1.32(1.08-1.63)$ & $318(44.9)$ & $1.20(0.93-1.54)$ \\
\hline$>37.0$ & 136(25.9) & $1.03(0.82-1.31)$ & $19(4.1)$ & $0.55(0.33-0.90)$ & $33(6.9)$ & $1.07(0.71-1.59)$ & $228(42.6)$ & $1.32(1.08-1.60)$ & $150(29.0)$ & $1.55(1.24-1.94)$ & $259(47.4)$ & $1.34(1.00-1.78)$ \\
\hline$p$ for trend & & 0.3567 & & 0.0033 & & 0.8943 & & 0.0049 & & $<0.0001$ & & 0.0147 \\
\hline \multicolumn{13}{|c|}{ Alcohol from rice wine $(\mathrm{g} / \mathrm{d})$} \\
\hline 0 & $966(22.8)$ & 1.0 & $251(6.5)$ & 1.0 & 229(5.9) & 1.0 & 1464(34.0) & 1.0 & $828(20.3)$ & 1.0 & 1793(40.4) & 1.0 \\
\hline$\leq 9.0$ & $182(22.7)$ & $0.98(0.80-1.20)$ & $34(4.6)$ & $0.66(0.45-0.96)$ & $38(5.2)$ & $0.78(0.54-1.12)$ & 269(33.6) & 0.94(0.79-1.12) & 148(19.3) & $0.91(0.74-1.12)$ & $348(42.0)$ & $1.17(0.92-1.48)$ \\
\hline$\leq 25.7$ & $213(21.7)$ & $1.02(0.85-1.23)$ & $28(3.1)$ & $0.49(0.33-0.74)$ & $48(5.3)$ & $0.86(0.61-1.20)$ & $358(35.8)$ & $1.07(0.92-1.25)$ & 208(21.5) & $1.11(0.92-1.33)$ & $422(41.5)$ & $1.28(1.03-1.59)$ \\
\hline$>25.7$ & $136(22.4)$ & 1.09(0.86-1.37) & $18(3.3)$ & $0.54(0.32-0.89)$ & $23(4.2)$ & $0.67(0.43-1.06)$ & 244(39.3) & $1.24(1.03-1.50)$ & 127(21.6) & $1.14(0.91-1.43)$ & 244(39.1) & $1.01(0.77-1.32)$ \\
\hline$p$ for trend & & 0.5419 & & $<0.0001$ & & 0.0587 & & 0.0400 & & 0.1856 & & 0.1770 \\
\hline \multicolumn{13}{|c|}{ All sources of alcohol $(\mathrm{g} / \mathrm{d})$} \\
\hline 0 & $652(21.2)$ & 1.0 & 198(7.0) & 1.0 & $162(5.8)$ & 1.0 & 1005(32.4) & 1.0 & $550(18.7)$ & 1.0 & 1242(38.6) & 1.0 \\
\hline$\leq 16.4$ & $283(24.0)$ & $1.05(0.88-1.25)$ & $54(5.1)$ & $0.61(0.44-0.85)$ & $67(6.3)$ & $1.02(0.75-1.38)$ & $417(35.1)$ & $1.14(0.98-1.33)$ & 218(19.5) & $1.00(0.83-1.21)$ & $506(41.5)$ & $0.95(0.76-1.17)$ \\
\hline$\leq 45.2$ & $259(21.7)$ & $0.94(0.78-1.13)$ & $43(3.9)$ & $0.46(0.32-0.65)$ & $49(4.5)$ & $0.66(0.47-0.94)$ & $436(35.9)$ & $1.19(1.02-1.38)$ & $258(22.0)$ & $1.18(0.99-1.41)$ & $537(43.1)$ & $1.32(1.07-1.64)$ \\
\hline$>45.2$ & $300(25.3)$ & 1.16(0.97-1.39) & $36(3.4)$ & $0.40(0.27-0.58)$ & $60(5.6)$ & $0.86(0.62-1.19)$ & $474(39.2)$ & $1.33(1.14-1.55)$ & $284(24.5)$ & $1.37(1.14-1.63)$ & $521(42.6)$ & $1.14(0.92-1.42)$ \\
\hline$p$ for trend & & 0.2925 & & $<0.0001$ & & 0.0926 & & 0.0002 & & 0.0005 & & 0.0517 \\
\hline
\end{tabular}

${ }^{*}$ Abnormal TG, HDL, Glucose and blood pressure, respectively. All models were adjusted for age at interview, BMI, education, marriage status, personal income, occupation, exercise, smoking status, tea consumption, and intake of meat, fish, soy food, fruits and vegetables. 

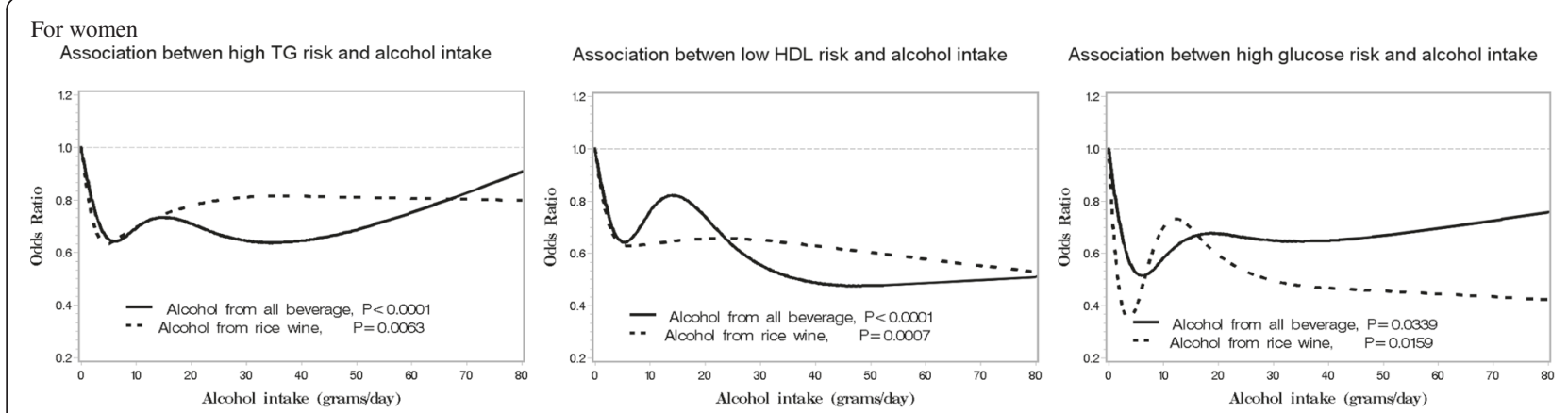

For men

Association betwen low HDL risk and alcohol intake
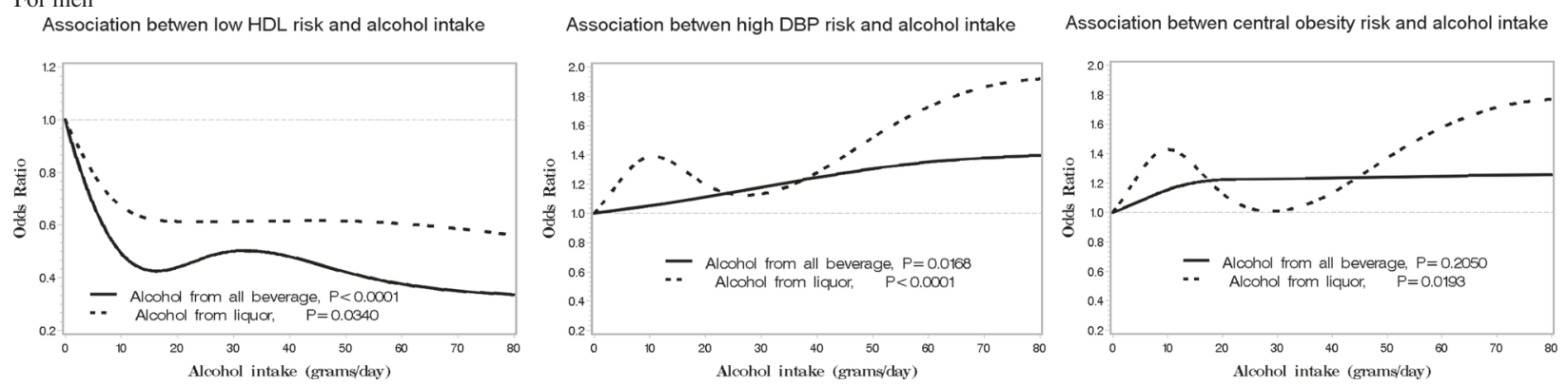

Figure 1 Association between alcohol consumption and MS components risk. Legend: Association between the MS components risk and various alcohol beverage intakes in 13,505 women and 6997 men in rural China; the NMSS during 2007-2008 and all p value were from linear trend test.

One of favorable effects of alcohol consumption is to decrease the risk of having low HDL-c levels [34]. We found that a dose-response relation between HDL-c levels and the quantity of beer, rice wine, liquor or combined alcoholic beverage consumption in both male and female participants. This result is consistent with early studies $[7,9,10,18,22,35]$. A linear association between alcohol and wine intake with the number of HDL particles has been documented [36]. However, we found the association was stronger among rice wine or beer drinkers than that of combined alcoholic beverage drinkers, suggesting that additional components polyphenols in wine other than alcohol might play an important role on lipid profiles, through its anti-inflammatory, insulin-sensitizing and vasodilatory properties [37]. It is possible that alcohol consumption (especially rice wine and beer) increased the hepatic production of apolipoproteins and TG lipase concentrations and decreased the removal of circulating HDL-c $[38,39]$.

In current study, we found rice wine consumption was related with constantly decreasing risk of high TG and high glucose in female drinkers, and also the risk of both high TG and high glucose was decreased rapidly if a woman drank all alcoholic beverage less than $10 \mathrm{~g} / \mathrm{d}$ and this protective effect was gradually leveled off if drinking more than $10 \mathrm{~g} / \mathrm{d}$. Our findings are consistent with that in previous studies in which they found light-tomoderate alcohol consumption may be associated with decreased triglycerides [19,40-42] and blood glucose $[22,43]$. The mechanism of this protective effect is plausible. Several studies suggested that the polyphenols enriched in red wine possess multiple benefits on MS and its components beyond alcohol through their anti-oxidant, anti-inflammatory, vascular-protective and insulin-sensitizing properties [22,37]. Resveratrol, a polyphenolic compound enriched in red wine, has recently attracted enormous attention because of combating the ageing process induced by nutrient excess [44]. Furthermore, Chinese rice wine contains large amount of polyphenol substance with strong ability of antioxidation [45], which is similar to that of red wine and may partially explain association of rice wine consumption and low risk of MS components among women.

Interestingly, protective effects in female drinkers were not found in male drinkers in current study. Conversely, we observed that alcoholic beverage may increase risk of high BP and central obesity among male drinkers, especially for liquor drinkers. This result is similar with several studies. In a meta-analysis, McFadden et al. reported that daily alcohol intake increased SBP by $2.7 \mathrm{mmHg}$ and DPB by $1.4 \mathrm{mmHg}$ [46]. Several prospective studies reported that no association of increasing risk of hypertension with light-to-moderate level of alcohol consumption in white men [15] but excessive alcohol consumption could lead to hypertension [16]. The difference of association of alcoholic 
beverage consumption and MS components between males and females might be due to unhealthy drinking patterns, including quantity and types of alcoholic beverage consumed [30,47]. Literature review showed high amount of alcohol intake remains harmful by elevating triglycerides [35,48]. Alcohol-induced hypertriglyceridemia, due to increased very-low-density lipoprotein secretion, impaired lipolysis and increased free fatty acid fluxes from adipose tissue to the liver. While light-to-moderate alcohol consumption may be associated with decreased triglycerides [19,40-42], reduce $30 \%$ of diabetes risk and the risk of having high blood glucose in male [22,43], and no association of increasing risk of hypertension in white men [15]. In agree with previous studies, male drinkers had 2-3 times of alcohol consumption and may lead to harmful effect of alcoholic beverage on MS components, compared with female drinkers in current study. Severe drinkers, no matter what type of alcoholic beverage consumed, may associate with obesity. We did not find association between any alcohol beverages and waist circumference in Chinese women because of their light-to-moderate alcohol consumption. It is consistent with previous study in Japan [49] and America [24]. But we found liquor was associated with waist circumference in men, which is in line with previous studies. Severe alcohol consumers $(\geq 50.0 \mathrm{~g} / \mathrm{d})$ had an increased risk of central obesity in middle-aged and elderly Chinese [43], especially in men [50,51] and in beer or liquor drinkers [50] because beer or liquor provides more energy than other types of alcoholic beverages and severe drinkers may lead to obesity because of excessive energy intake, lipid oxidation and fat accumulation [52]. A recent study demonstrated that alcohol also inhibits ghrelin secretion, resulting in appetite stimulation among men [53]. Moreover, type of alcoholic beverage may play an impotent role in association with MS components. Kao et al. found that severe liquor drinking was shown to increase the risk of type II diabetes [54], whereas similar amount of beer or wine did not, implying this effect is related to types of alcoholic beverage. Given rice wine has protective effect on MS, high TG and high glucose, unhealthy drinking pattern, especially for sever drink, could attenuate this effect among male participants in our study.

The strengths of this study include using a large pool of population-based data, extensive information on confounders, and standard methods and vigorous quality control to measure all variables. The study does, however, have several limitations and considerations. First, given the cross-sectional design, we could not draw any causal inference regarding the association of alcohol consumption with MS components. Therefore, future prospective studies are required to confirm our findings.
Second, alcohol consumption status was based on the results of self-reported questionnaires, which might have recall bias. Finally, and we cannot do more significant analysis by combining years and amount of drinking together because 'years of drinking' is not available.

\section{Conclusions}

In summary, alcohol consumption is associated with a lower prevalence of MS among Chinese women. A favorable effect on TG and glucose is found among rice wine consumers in female and light-to-moderate combined alcoholic consumers. Any type of alcohol beverage, including beer, liquor, and rice wine might decrease the risk of lower HDL-c in both genders. But beneficial effect of alcoholic consumption was offsetting by severe drinkers in men, which lead to no association between alcohol consumption and MS in our study. Our data provide an evidence that rice wine and healthy drinking patterns have a beneficial effect on serum lipids and serum glucose, and has better protective effects than any other alcoholic beverages on MS components among Chinese women.

\section{Abbreviations}

MS: Metabolic syndrome; NMSS: Nantong metabolic syndrome study; TG: Triglycerides; HDL-c: High-density lipoprtein cholesterol; BP: Blood pressure; SBP: Systolic blood pressure, DBP, Diastolic blood pressure; IDF: International diabetes federation; CDC: the Nantong centers for disease control; BMI: Body mass index; ORs: Odds ratios; Cls: Confidence intervals.

\section{Competing interests}

The authors declare that they have no competing interests.

\section{Authors' contributions}

JX drafted the manuscript, conducted the analyses of the data, and revised the manuscript for important intellectual content and approved the final version for submission. JPH provided statistical support, contributed to the design of the study and critically reviewed the manuscript. GFX supervised the statistical plan and revised the article for important intellectual content and approved the final version for submission. DXC, GYW, and MZ contributed to data collection and analysis. YS revised the article for important intellectual content and approved the final version for submission. HC conceived and designed the main study and is the guarantor of this work and, as such, had full access to all the data in the study and takes responsibility for the integrity of the data and the accuracy of the data analysis. All authors read and approve the final manuscript.

\section{Acknowledgment and funding}

We deeply appreciate the participants of the NMSS in Nantong, China. This work was supported by the National Natural Science Foundation of China (31000539), the Ministry of Science and Technology of China (2006 BA1 01A 01), and the Department of Science and Technology of Nantong (BK2011059, S2010042). The funders had no role in study design, data collection and analysis, decision to publish or preparation of the manuscript.

\section{Author details}

${ }^{1}$ Department of Epidemiology and Medical Statistics, School of Public Health, Nantong University, Nantong, Jiangsu, China. ${ }^{2}$ Department of Chronic Disease and Prevention Center, Center for Disease Control and Prevention of Nantong, Nantong, Jiangsu, China. ${ }^{3}$ Department of Nutrition and Food Science, School of Public Health, Nantong University, Nantong, Jiangsu, China. 
Received: 6 November 2014 Accepted: 14 February 2015 Published online: 28 February 2015

\section{References}

1. Chen CC, Lin WY, Li Cl, Liu CS, Li TC, Chen YT, et al. The association of alcoholic consumption with metabolic syndrome and its individual components: the Taichung community health study. Nutr Res. 2012;32:24-9.

2. Hanson RL, Imperatore G, Bennett PH, Knowler WC. Components of the "metabolic syndrome" and incidence of type 2 diabetes. Diabetes. 2002:51:3120-7.

3. Gami AS, Witt BJ, Howard DE, Erwin PJ, Gami LA, Somers VK, et al. Metabolic syndrome and risk of incummerident cardiovascular events and death: a systematic review and meta-analysis of longitudinal studies. J Am Coll Cardiol. 2007;49:403-14.

4. Ford ES, Giles WH, Dietz WH. Prevalence of the metabolic syndrome among US adults: findings from the third National Health and Nutrition Examination Survey. JAMA. 2002;287:356-9.

5. Alberti KG, Zimmet P, Shaw J. Metabolic syndrome-a new world-wide definition. A consensus statement from the international diabetes federation. Diabet Med. 2006;23:469-80.

6. Liese AD, Mayer-Davis EJ, Haffner SM. Development of the multiple metabolic syndrome: an epidemiologic perspective. Epidemiol Rev. 1998;20:157-72.

7. Maria TBL, Maira BR, Carmen SO, Martin GL, Alejandro FM, Alfredo G, et al. Different types of alcoholic beverages and incidence of metabolic syndrome and its components in a Mediterranean cohort. Clin Nutr. 2013;32:797-804.

8. Alkerwi A, Boutsen M, Vaillant M, Barre J, Lair ML, Albert A, et al. Alcohol consumption and the prevalence of metabolic syndrome: A meta-analysis of observational studies. Atherosclerosis. 2009;204:624-35.

9. Seike N, Noda M, Kadowaki T. Alcohol consumption and risk of type 2 diabetes mellitus in Japanese: a systematic review. Asia Pac J Clin Nutr. 2008:17:545-51.

10. Yin RX, Pan SL, Chen H, Yang HL, Wu H, Chen YM, et al. Diet, alcoho consumption, and serum lipid levels of the middle-aged and elderly in the Guangxi Bai Ku Yao and Han populations. Alcohol. 2008;42:219-29.

11. Taylor B, Irving HM, Baliunas D, Roerecke M, Patra J, Mohapatra S, et al. Alcohol and hypertension: gender differences in dose-response relationships determined through systematic review and meta-analysis. Addiction. 2009:104:1981-90.

12. Sesso HD, Cook NR, Buring JE, Manson JE, Gaziano JM. Alcohol consumption and the risk of hypertension in women and men. Hypertension. 2008:51:1080-7.

13. Schroder H, Morales-Molina JA, Bermejo S, Barral D, Mandoli ES, Grau M, et al. Relationship of abdominal obesity with alcohol consumption at population scale. Eur J Nutr. 2007:46:369-76.

14. Riserus $U$, Ingelsson E. Alcohol intake, insulin resistance, and abdominal obesity in elderly men. Obesity. 2007;15:1766-73.

15. Yoon YS, Oh SW, Baik HW, Park HS, Kim WY. Alcohol consumption and the metabolic syndrome in Korean adults: the 1998 Korean National Health and Nutirtion Examination Survey. Am J Clin Nutr. 2004:80:217-24.

16. Lee KS, Park CY, Meng KH, Bush A, Lee SH, Lee WC, et al. The association of cigarette smoking and alcohol consumption with other cardiovascular risk factors in men from Seoul, Korea. Ann Epidemiol. 1998;8:31-8.

17. Zhang M, Zhao J, Tong W, Wang A, Huang G, Zhang Y. Associations between metabolic syndrome and its components and alcohol drinking. Exp Clin Endocrinol Diabetes. 2011;119:509-12.

18. Sayon-Orea C, Martinez-Gonzalez MA, Bes-Rastrollo M. Alcohol consumption and body weight: a sustematic review. Nutr Rev. 2011;69:419-31.

19. Suh I, Shaten BJ, Cutler JA, Kuller LH. Alcohol use and mortality from coronary heart disease: the role of high-density lipoprotein cholesterol. The multiple risk factor intervention trial research group. Ann Intern Med. 1992;116:881-7.

20. Linn S, Carroll M, Johnson C, Fulwood R, Kalsbeek W, Briefel R. High-density lipoprotein cholesterol and alcohol consumption in US white and black adults: data from NHANES II. Am J Public Health. 1993;83:811-6.

21. Stoutenberg $M$, Lee DC, Sui XM, Hooker S, Horigian V, Perrino T, et al. Prospective study of alcohol consumption and the incidence of the metabolic syndrome in US men. Br J Nutr. 2013;110:901-10.

22. Shi L, Shu XO, Li H, Cai H, Liu QL, Zheng W, et al. Physical activity, smoking, and alcohol consumption in association with incidence of type 2 diabetes among middle-aged and elderly Chinese men. PLoS One. 2013;8:e77919.
23. Ley SH, Sun Q, Jimenez MC, Rexrode KM, Manson JE, Jensen MK, et al. Association between alcohol consumption and plasma fetuin-A and its contribution to incident type 2 diabetes in women. Diabetologia. 2014;57:93-101.

24. Koppes LL, Dekker JM, Hendriks HF, Bouter LM, Heine RJ. Moderate alcohol consumption lowers the risk of type 2 diabetes: a meta-analysis of prospective observational studies. Diabetes Care. 2005;28:719-25.

25. Cai H, Huang JP, Xu GF, Yang ZL, Liu M, Mi YP, et al. Prevalence and determinants of metabolic syndrome among women in Chinese rural areas. PLoS One. 2012;7:e36936

26. Shu XO, Hatch MC, Mills J, Clemens J, Susser M. Maternal smoking, alcohol drinking, caffeine consumption, and fetal growth: results from a prospective study. Epidemiology. 1995;6:115-20.

27. Alberti KGMM, Eckel RH, Grundy SM, Zimmet PZ, Cleeman JL, Donato KA, et al. Harmonizing the metabolic syndrome: a joint interim statement of the International Diabetes Federation Task Force on Epidemiology and Prevention; National Heart, Lung, and Blood Institute; American Heart Association; World Heart Federation; International Atherosclerosis Society; and International Association for the Study of Obesity. Circulation. 2009;120 (16):1640-5.

28. Baik I, Shin C. Prospective study of alcohol consumption and metabolic syndrome. Am J Clin Nutr. 2008;87:1455-63.

29. Fan JG, Cai XB, Li L, Li XJ, Dai F, Zhu J. Alcohol consumption and metabolic syndrome among Shanghai adults: a randomized multistage stratified cluster sampling investigation. World J Gastroenterol. 2008;14:2418-24.

30. Shin MH, Kweon SS, Choi JS, Rhee JA, Nam HS, Jeong SK, et al. Average volume of alcohol consumed, drinking patterns, and metabolic syndrome in older Korean adults. J Epidemiol. 2013;23:122-31.

31. Freiberg MS, Cabral HJ, Heeren TC, Vasan RS, Curtis ER. Alcohol consumption and the prevalence of the metabolic syndrome in the U.S. A cross-sectional analysis of data from the Third National Health and Nutrition Examination Survey. Diabetes Care. 2004;27:2954-9.

32. Park YW, Zhu S, Palaniappan L, Heshka S, Carnethon MR, Heymsfield SB. The metabolic syndrome: prevalence and associated risk factor findings in the US population from the Third National Health and Nutrition Examination Survey, 1988-1994. Arch Intern Med. 2003;163:427-36.

33. Fujita N, Takei Y. Alcohol consumption and metabolic syndrome. Hepatology Research. 2011;41:287-95.

34. Ashen MD, Blumenthal RS. Clinical practice. Low HDL cholesterol levels. N Engl J Med. 2005;353:1252-60.

35. Rimm EB, Williams P, Fosher K, Criqui M, Stampfer MJ. Moderate alcohol intake and lower risk of coronary heart disease: meat-analysis of effects on lipids and haemostatic factors. BMJ. 1999;319:1523-8.

36. Rimm EB, Chan J, Stampfer MJ, Colditz GA, Willett WC. Prospective study of cigarette smoking, alcohol use, and the risk of diabetes in men. BMJ. 1995;310:555-9.

37. Liu L, Wang Y, Lam KSL, Xu AM. Moderate wine consumption in the prevention of metabolic syndrome and its related medical complications. Endocrine, Metabolic \& Immune Disorders. 2008:8:89-98.

38. Moore RD, Smith CR, Kwiterovich PO, Pearson TA. Effect of low-dose alcohol use versus abstention on apolipoproteins A-I and B. Am J Med. 1988;84:884-90.

39. Hannuksela M, Marcel YL, Kesaniemi YA, Savolainen MJ. Reduction in the concentration and activity of plasma cholesteryl ester transfer protein by alcohol. J Lipid Res. 1992;33:737-44.

40. Facchini F, Chen Yl, Reaven GM. Light-to-moderate alcohol intake is association with enhanced insulin sensitivity. Diabetes Care. 1994;17:115-9.

41. Gaziano JM, Buring JE, Breslow JL, Goldhaber SZ, Rosner B, VanDenburgh M, et al. Moderate alcohol intake, increased levels of high-density lipoprotein and its subfractions, and decreased risk of myocardial infarction. N Engl J Med. 1993;329:1829-34.

42. Mayer EJ, Newman B, Quesenberry Jr CP, Friedman GD, Selby JV. Alcohol consumption and insulin concentrations. Role of insulin in associations of alcohol intake with high-density lipoprotein cholesterol and triglycerides. Circulation. 1993:88:2190-7.

43. Jin L, Huang Y, Bi YF, Zhao LB, Xu M, Xu Y, et al. Association between alcohol consumption and metabolic syndrome in 19,215 middle-aged and elderly Chinese. Diabetes Res Clin Pract. 2011;92:386-92.

44. Baur JA, Sinclair DA. Therapeutic potential of resveratrol: the in vivo evidence. Nat Rev Drug Discov. 2006;5:493-506.

45. Zheng XX, Li Y, Xu WF, Fang YQ, Ran YZ. Research on the antioxidation of yellow rice wine. Liquor-Making Sci Technol. 2009;10:57-9. 
46. McFadden CB, Brensinger CM, Berlin JA, Townsend RR. Systematic review of the effect of daily alcohol intake on blood pressure. Am J Hypertens. 2005;18:276-86.

47. Fan AZ, Russell M, Naimi T, Li Y, Liao Y, Jiles R, et al. Patterns of alcohol consumption and the metabolic syndrome. J Clin Endocrinol Metab. 2008;93:3833-8.

48. Klop B, Do Rego AT, Cabezas MC. Alcohol and plasma triglycerides. Current opinion in lipidology. 2013;24:321-6.

49. Wakabayashi I. Association between alcohol drinking and metabolic syndrome in Japanese male workers with diabetes mellitus. J Atberoscler Tbromb. 2011;18:684-92

50. Slattery ML, McDonald A, Bild DE, Caan BJ, Hilner JE, Jacobs Jr DR, et al. Association of body fat and its distribution with dietary intake, physical activity, alcohol, and smoking in blacks and whites. Am J Clin Nutr. 1992:55:943-9.

51. Tolstrup JS, Heitmann BL, Tjonneland AM, Overvad OK, Sorensen TI, Gronbaek MN. The relation between drinking pattern and body mass index and waist and hip circumference. Int J Obes. 2005;29:490-7.

52. Suter PM. Is alcohol consumption a risk factor for weight gain and obesity? Crit Rev Clin Lab Sci. 2005;42:197-227.

53. Calissendorff J, Danielsson O, Brismar K, Rojdmark S. Inhibitory effect of alcohol on ghrelin secretion in normal man. Eur J Endocrinol. 2005;152:743-7.

54. Kao WH, Puddey IB, Boland LL, Watson RL, Brancati FL. Alcohol consumption and the risk of type 2 diabetes mellitus: atherosclerosis risk in communities study. Am J Epidemiol. 2001;154:748-57.

\section{Submit your next manuscript to BioMed Central and take full advantage of:}

- Convenient online submission

- Thorough peer review

- No space constraints or color figure charges

- Immediate publication on acceptance

- Inclusion in PubMed, CAS, Scopus and Google Scholar

- Research which is freely available for redistribution 